\title{
Beliefs on Mandatory Influenza Vaccination of Health Care Workers in Nursing Homes: A Questionnaire Study from the Netherlands
}

\author{
Ingrid Looijmans-van den Akker, MD, PhD, ${ }^{*}$ Badyr Marsaoui, MD, ${ }^{*}$ Eelko Hak, MSc, PhD, ${ }^{* \dagger}$ \\ and Johannes J. M. van Delden, MD, PhD*
}

OBJECTIVES: To assess whether nursing homes (NHs) made organizational improvements to increase influenza vaccination rates in healthcare workers (HCWs) and to quantify the beliefs of $\mathrm{NH}$ administrators on the arguments used in favor of implementation of mandatory influenza vaccination of HCWs.

DESIGN: Anonymous questionnaire study.

SETTING: Dutch NHs.

PARTICIPANTS: Dutch NH administrators.

MEASUREMENTS: Influenza vaccination rates in $\mathrm{NH}$ residents and NH HCWs, organizational aspects of influenza vaccination of HCWs, and agreement of respondents with arguments in favor of implementation of mandatory influenza vaccination in HCWs.

RESULTS: Of the 310 distributed questionnaires, 185 were returned (response rate $59.7 \%$ ). The average vaccination rate in $\mathrm{NH}$ HCWs was $18.8 \%$ and in $\mathrm{NH}$ residents was $91.6 \%$. In all, $126(68.1 \%)$ NHs had a written policy, $161(87.0 \%)$ actively requested that their employees be immunized, and $161(87.0 \%)$ offered information to HCWs in any way. Despite the fact that the majority of $\mathrm{NH}$ administrators $(>69 \%$ ) agreed with all arguments in favor of implementation of mandatory influenza vaccination, only a minority $(24.3 \%)$ agreed that mandatory vaccination should be implemented if voluntary vaccination fails to reach sufficient vaccination rates.

CONCLUSION: Despite the low vaccination rate of $\mathrm{NH}$ HCWs, most $\mathrm{NH}$ administrators did not support mandatory influenza vaccination of NH HCWs. J Am Geriatr Soc 57:2253-2256, 2009.

From the * Julius Center for Health Sciences and Primary Health Care, University Medical Center Utrecht, Utrecht, The Netherlands; and 'Department of Epidemiology, University Medical Center Groningen, Groningen, The Netherlands.

Address correspondence to I. Looijmans-van den Akker, University Medical Center Utrecht, Julius Center for Health Sciences and Primary Health Care, HP 6.131 (room S4.149), P O Box 85500, 3508 GA Utrecht, The Netherlands. E-mail: i.looijmans@umcutrecht.nl

DOI: 10.1111/j.1532-5415.2009.02560.x
Key words: healthcare workers; mandatory; influenza vaccination

$S^{\mathrm{n}}$ tudies have demonstrated that influenza vaccination of healthcare workers (HCWs) reduces mortality and morbidity in nursing home (NH) residents. ${ }^{1-3}$ In addition, it reduces the medical and non-healthcare costs related to influenza outbreaks and influenza-associated absenteeism of NH HCWs. ${ }^{4,5}$ The Dutch association of $\mathrm{NH}$ physicians published a guideline in 2004 in which it recommended that all HCWs in NHs be immunized against influenza, followed by a national recommendation by the Health Council of the Netherlands in 2007.6,7 Despite these official recommendations and the efforts of institutions to increase vaccination rates, vaccination rates in HCWs remain low, less than $25 \%$ in Europe. 8,9

Persistently low vaccination rates in HCWs have led to debate between international scientists on implementing mandatory influenza vaccination. Those who favor mandatory vaccination cite the professional responsibility of HCWs not to do harm and their duty to support the collective efforts of the institution at which they work, which justifies some infringement of their personal autonomy. ${ }^{10-12}$

Before the 2008/09 influenza season, a questionnaire study was conducted following a previous study. ${ }^{13}$ It was hypothesized that NHs made organizational improvements to increase influenza vaccination rates in HCWs. In addition, the extent to which $\mathrm{NH}$ administrators agree with the arguments in favor of mandatory influenza vaccination for NH HCWs was quantified.

\section{METHODS}

\section{Participants and Methods}

In October 2005, a questionnaire was sent to the administrators of all Dutch NHs $(\mathrm{N}=335) .{ }^{13}$ The following items were assessed: influenza vaccination rates in $\mathrm{NH}$ residents 
and NH HCWs in the preceding season (2004/05 season), whether the institution had a written policy on influenza vaccination for HCWs, what the current offering policy was (active request, employee initiative, or none), and whether HCWs were offered information on influenza vaccination. In October and November 2008, similar questions were asked, supplemented with items concerning arguments used in the literature for implementation of mandatory influenza vaccination in NH HCWs. ${ }^{10}$ This self-administered, 11item anonymous questionnaire was sent to the administrators of all Dutch NHs $(\mathrm{N}=310)$. The total number of NHs was less than in 2005 because of closing and merging of 25 NHs. Reminders were sent after 2 and 4 weeks.

Items concerning implementation of mandatory influenza vaccination were that influenza vaccination of $\mathrm{NH}$ HCWs is effective in reducing mortality and morbidity of $\mathrm{NH}$ residents, influenza vaccination of NH HCWs is effective in reducing the costs related to influenza, $\mathrm{NH} \mathrm{HCWs}$ have a special responsibility in preventing influenza transmission to $\mathrm{NH}$ residents, administrators have a moral responsibility to implement voluntary influenza vaccination of NH HCWs, and mandatory influenza vaccination of $\mathrm{NH}$ HCWs should be implemented if voluntary vaccination fails to reach a sufficient vaccination rate. Respondents were asked to state to which extent they agreed or disagreed with these propositions on a 5 -point Likert scale ranging from strongly agree to strongly disagree. Finally respondents were asked whether they had any supplementary comments. For the English translation of the questionnaire see Appendix 1.

\section{Statistical Analysis}

Data were analyzed using SPSS for windows (version 14.0, SPSS, Inc., Chicago, IL). The question on the current offering policy was dichotomized as active request versus employee initiative or none. The question on information offered to NH HCWs was dichotomized as any form of information versus no information offered. Questions on the 5-point Likert scale were used to compare respondents who strongly agreed or agreed with those who were uncertain (did not agree or did not disagree), disagreed, or strongly disagreed. Descriptive statistics such as interquartile range were used to relate the vaccination rate in $\mathrm{NH}$ HCWs to other variables. To determine which factors were associated with influenza vaccination rate, univariate associations were assessed. Mean differences and their $95 \%$ confidence intervals (CIs) were reported as measures of associations.

\section{RESULTS}

\section{Study Population}

Of 310 distributed questionnaires, 185 were returned (response rate of $59.7 \%$ ). The average vaccination rate in $\mathrm{NH}$ HCWs was $18.8 \%$ (95\% CI $=16.5-21.1 \%$, range $0-80 \%$, median value $15 \%$, interquartile range $=10-25 \%$ ) and $91.6 \%$ in $\mathrm{NH}$ residents $(95 \% \mathrm{CI}=89.7-93.5 \%)$.

\section{Organizational Factors}

In all, $161(87.0 \%) \mathrm{NHs}$ actively requested that their employees be immunized, $161(87.0 \%)$ offered information to
HCWs in any way, and 126 (68.1\%) had a written policy. Actively requesting $\mathrm{NH}$ that $\mathrm{HCW}$ se immunized and informing NH HCWs about influenza vaccination were associated with significantly higher mean vaccination rates in $\mathrm{NH}$ HCWs (Table 2). NHs with HCW vaccination rates in the first quartile $(<10 \%)$ had lower percentages of having a written policy than those in the second to fourth quartile (percentages were $58.6 \%, 75.0 \%, 75.5 \%$, and $72.2 \%$ for NH HCW vaccination rate quartiles $1,2,3$, and 4 , respectively). These NHs also had the lowest percentages of actively requesting that HCWs be immunized $(74.6 \%$, $97.1 \%, 95.5 \%$, and $92.1 \%$ ) and offering information to HCWs in any way $(74.6 \%, 97.1 \%, 95.5 \%$, and $92.1 \%)$.

\section{NH Administrator Beliefs}

The majority $(>69 \%$ ) of $\mathrm{NH}$ administrators agreed with the first four statements described above (Table 1), although only $24.3 \%$ of $\mathrm{NH}$ administrators agreed with the statement that mandatory influenza vaccination of $\mathrm{NH} \mathrm{HCWs}$ should be implemented if voluntary vaccination programs failed to reach a sufficient vaccination rate. NHs in which the administrators agreed with the following statements had a significantly higher mean vaccination rate of $\mathrm{NH}$ HCWs: Influenza vaccination is effective in reducing the costs related to influenza. NH HCWs have a special responsibility in preventing influenza transmission to $\mathrm{NH}$ residents. Administrators have a moral responsibility to implement voluntary influenza vaccination of NH HCWs (Table 2). In addition, some comments against the implementation of mandatory vaccination were encountered. The arguments used were the infringement of autonomy of NH HCWs and religious backgrounds. There were also comments of respondents who stopped offering NH HCWs voluntary influenza vaccination, because it did not result in a relevant increase in vaccination rates. One of the respon-

\begin{tabular}{|c|c|}
\hline Statement & $\begin{array}{c}\text { NH Administrators Who } \\
\text { Agreed or Strongly Agreed, } \\
n(\%)\end{array}$ \\
\hline $\begin{array}{l}\text { Influenza vaccination of NH HCWs is } \\
\text { effective in reducing mortality and morbidity } \\
\text { of the NH residents. }\end{array}$ & $153(82.7)$ \\
\hline $\begin{array}{l}\text { Influenza vaccination of NH HCWs is } \\
\text { effective in reducing the costs related to } \\
\text { influenza. }\end{array}$ & 128 (69.2) \\
\hline $\begin{array}{l}\text { NH HCWs have a special responsibility in } \\
\text { preventing influenza transmission to } \mathrm{NH} \\
\text { residents. }\end{array}$ & $160(86.5)$ \\
\hline $\begin{array}{l}\text { The administrators have a moral } \\
\text { responsibility to implement voluntary } \\
\text { influenza vaccination of NH HCWs. }\end{array}$ & 154 (83.2) \\
\hline $\begin{array}{l}\text { Mandatory influenza vaccination of NH } \\
\text { HCWs should be implemented if voluntary } \\
\text { vaccination fails to reach a sufficient } \\
\text { vaccination rate. }\end{array}$ & 45 (24.3) \\
\hline
\end{tabular}


Table 2. Effects of Policy Determinants and Beliefs on Mean Influenza Vaccination Rates in Nursing Home (NH) Healthcare workers (HCWs) $(\mathrm{N}=185)$

\begin{tabular}{|c|c|c|c|}
\hline \multirow[b]{2}{*}{ Factors } & \multicolumn{2}{|c|}{ HCWs vaccinated $n(\%)$} & \multirow{2}{*}{$\begin{array}{c}\text { Mean Difference } \\
\text { (95\% Confidence Interval) }\end{array}$} \\
\hline & Yes & No & \\
\hline Offering information to NH HCWs in any way & $161(19.9)$ & $23(10.7)$ & $9.2(2.2-16.2)$ \\
\hline Having a written policy & $126(19.5)$ & $56(16.5)$ & $2.9(-2.0-7.9)$ \\
\hline $\begin{array}{l}\text { Agreement with the statement that influenza vaccination of NH HCWs is } \\
\text { effective in reducing mortality and morbidity of the NH residents }\end{array}$ & $153(19.3)$ & $32(16.4)$ & $2.8(-3.3-9.0)$ \\
\hline $\begin{array}{l}\text { Agreement with the statement that influenza vaccination of NH HCWs is } \\
\text { effective in reducing the costs related to influenza }\end{array}$ & $128(20.6)$ & $57(14.7)$ & $5.9(1.0-10.8)$ \\
\hline $\begin{array}{l}\text { Agreement with the statement that NH HCWs have a special responsibility } \\
\text { in preventing influenza transmission to NH residents }\end{array}$ & $160(20.3)$ & $25(8.2)$ & $12.1(8.1-16.1)$ \\
\hline $\begin{array}{l}\text { Agreement with the statement that the administrators have a moral } \\
\text { responsibility to implement voluntary influenza vaccination of NH HCWs }\end{array}$ & $154(20.0)$ & $29(11.4)$ & $8.6(2.2-15.1)$ \\
\hline
\end{tabular}

dents stated that programs to stimulate increase of vaccination rates in NH HCWs were a waste of time.

\section{DISCUSSION}

Influenza vaccination rates in $\mathrm{NH} \mathrm{HCW}$ increased only slightly despite organizational improvements and increasing awareness of institutions of the need for influenza vaccination. Most NHs agreed with all arguments in favor of implementation of mandatory influenza vaccination, but most NHs were against mandatory vaccination if voluntary programs failed to reach a sufficient vaccination rate. This is the first study to quantify to what extent $\mathrm{NH}$ administrators agreed with the arguments in favor of implementation of mandatory influenza vaccination.

Some potential limitations of the study need to be addressed. The response rate of $59.7 \%$ was reasonable and comparable with that in previous studies, ${ }^{13-15}$ but it was an anonymous questionnaire study, and responders could not be compared with all Dutch NHs. Therefore, response bias cannot be excluded, and overestimation of the vaccination rate in NH HCWs and underestimation of the organizational improvements and agreement with the arguments in this study is possible. ${ }^{14,16,17}$ Nevertheless, similar vaccination rates were found in $\mathrm{NH}$ residents as in previous studies. ${ }^{13,15}$

Influenza vaccination rates in $\mathrm{NH} \mathrm{HCW}$ s were higher than in a previous study performed in 2005 (19\% vs $10 \%)$. Furthermore a higher percentage was observed to have a written policy ( $68 \%$ vs $45 \%)$ actively requesting that $\mathrm{NH}$ HCWs be immunized $(87 \%$ vs $72 \%$ ) and informing $\mathrm{NH}$ HCWs in any way $(87 \%$ vs $74 \%) .{ }^{13}$ It is likely that these organizational improvements contributed to the absolute increase of $9 \%$ in influenza vaccination rates in $\mathrm{NH}$ HCWs. Actively requesting that $\mathrm{NH} \mathrm{HCWs} \mathrm{be} \mathrm{immunized} \mathrm{and} \mathrm{in-}$ forming NH HCWs about influenza vaccination were also associated with significantly higher mean vaccination rates in NH HCWs (Table 2). Comparable associations were found in a previous study. ${ }^{13}$ It was also found that the NHs in the second through fourth quartiles of NH HCW vaccination rates had steady high percentages in actively re- questing that HCWs be immunized and in offering information to HCWs in any way. Above this threshold, standard measures seem to be ineffective in further increasing the vaccination rate of $\mathrm{NH} \mathrm{HCWs.}$

The question remains as to when influenza vaccine rates in NH HCWs can be considered high enough. A previously performed mathematical modeling study indicated that a threshold for herd immunity does not exist. ${ }^{18}$ Therefore, vaccination of additional $\mathrm{NH}$ HCWs protects an additional fraction of $\mathrm{NH}$ residents (number needed to vaccinate to prevent 1 infection $=7$ ). So the influenza vaccination rate of $\mathrm{NH}$ HCWs and the increase of it found in this study are of some value but should be improved further. Most studies that have evaluated the effectiveness of promotional campaigns in HCWs were of limited quality, and results varied. ${ }^{4}$ If future studies prove that voluntary programs are ineffective at increasing the influenza vaccination rate in NH HCWs, implementation of mandatory influenza vaccination might be an option, ${ }^{10}$ but a great majority of respondents did not agree with the statement that mandatory influenza vaccination of $\mathrm{NH}$ HCWs should be implemented if voluntary vaccination failed to reach a sufficient vaccination rate (Table 1).

$\mathrm{NH}$ administrators supported the arguments in favor of implementing mandatory vaccination, but they did not support implementing this measure. It might be that they find the arguments against implementation of mandatory vaccination, such as the autonomy of $\mathrm{NH} \mathrm{HCWs}$, more important than all arguments in favor of implementing mandatory vaccination.

\section{ACKNOWLEDGMENTS}

Conflict of Interest: The editor in chief has reviewed the conflict of interest checklist provided by the authors and has determined that the authors have no financial or any other kind of personal conflicts with this paper. Neither author received any funding for it.

Author Contributions: All authors contributed to the study concept and design, the acquisition of subjects and 
data, analysis and interpretation of data, and preparation of the manuscript.

\section{Sponsor's Role: No sponsor.}

\section{REFERENCES}

1. Hayward AC, Harling R, Wetten S et al. Effectiveness of an influenza vaccine programme for care home staff to prevent death, morbidity, and health service use among residents: Cluster randomised controlled trial. BMJ 2006;333:1241-1246.

2. Carman WF, Elder AG, Wallace LA et al. Effects of influenza vaccination of health-care workers on mortality of elderly people in long-term care: A randomised controlled trial. Lancet 2000;355:93-97.

3. Potter J, Stott DJ, Roberts MA et al. Influenza vaccination of health care workers in long-term-care hospitals reduces the mortality of elderly patients. J Infect Dis 1997;175:1-6.

4. Burls A, Jordan R, Barton P et al. Vaccinating healthcare workers against influenza to protect the vulnerable-Is it a good use of healthcare resources? A systematic review of the evidence and an economic evaluation. Vaccine 2006;24:4212-4221.

5. Saxen H, Virtanen M. Randomized, placebo-controlled double blind study on the efficacy of influenza immunization on absenteeism of health care workers. Pediatr Infect Dis J 1999;18:779-783.

6. Cools HJ, van Essen GA. Practice guideline 'Influenza prevention in nursing homes and care homes', issued by the Dutch Society of Nursing Home Specialists [in Dutch]. Ned Tijdschr Geneeskd 2005;149:213.

7. Influenza Vaccination: Revision of the Indication. Publication no. 2007/09 The Hague, The Netherlands: Health Council of the Netherlands, 2007.

8. Kroneman M, Paget WJ, van Essen GA. Influenza vaccination in Europe: An inventory of strategies to reach target populations and optimise vaccination uptake. Euro Surveill 2003;8:130-138.

9. Blank PR, Schwenkglenks M, Szucs TD. Influenza vaccination coverage rates in five European countries during season 2006/07 and trends over six consecutive seasons. BMC Public Health 2008;8:272.

10. van Delden JJ, Ashcroft R, Dawson A et al. The ethics of mandatory vaccination against influenza for health care workers. Vaccine 2008;26:5562-5566.

11. Helms CM, Polgreen PM. Should influenza immunisation be mandatory for healthcare workers? Yes. BMJ 2008;337:a2142.

12. van den Hoven MA, Verweij MF. Should we promote influenza vaccination of health care workers in nursing homes? Some ethical arguments in favour of immunization. Age Ageing 2003;32:487-489.

13. Looijmans-van den Akker I, van Delden JJ, Hak E. Uptake of influenza vaccination in Dutch nursing home personnel following national recommendations. J Am Geriatr Soc 2007;55:1486-1487.

14. Sibbald B, Addington-Hall J, Brenneman D et al. Telephone versus postal surveys of general practitioners: Methodological considerations. Br J Gen Pract 1994;44:297-300.

15. Verweij M, van den Hoven HM. Influenza vaccination rates and informed consent in Dutch nursing homes: Survey of nursing home physicians. BMJ 2002;324:328.

16. Armstrong D, Ashworth $\mathrm{M}$. When questionnaire response rates do matter: A survey of general practitioners and their views of NHS changes. Br J Gen Pract 2000;50:479-480.

17. Ashworth M. When response rates do matter. BMJ 2001;322:675.

18. van den Dool C, Bonten MJ, Hak E et al. The effects of influenza vaccination of health care workers in nursing homes: Insights from a mathematical model. PLoS Med 2008;5:e200.

\begin{tabular}{l} 
Appendix 1. English Translation of the Questionnaire \\
\hline 1. What percentage of the residents in \\
your nursing home was vaccinated \\
against influenza last year (autumn \\
2007)?
\end{tabular}

\section{What percentage of HCWs (health}

$\%$

care workers) in your nursing home was

vaccinated against influenza last year

(autumn 2007)?

3. Does your nursing home currently

have a written policy on influenza

vaccination HCWs?

\section{Appendix 1. (Contd.)}

4. What is the current policy on offering influenza vaccination to HCWs in your nursing home?

$\square$ We actively request that HCWs get vaccinated against influenza.

$\square$ HCWs can only get vaccinated on their own initiative.

$\square$ We do not offer influenza vaccination to the HCWs.

5. How are HCWs currently informed about influenza vaccination? (more than one answer allowed)

$\square$ By personal written information (e.g. invitation letters, leaflets)

$\square$ By collective written information (e.g. posters)

$\square$ By group oral information (e.g. information meeting)

$\square$ Other, namely ...

6. Do the administrators of your nursing home agree with the statement that influenza vaccination of HCWs is effective in reducing mortality and morbidity of the residents?

$\square$ Strongly agree
$\square$ Agree
$\square$ Do not agree/Do not disagree
$\square$ Disagree
$\square$ Strongly disagree

7. Do the administrators of your nursing home agree with the statement that influenza vaccination of HCWs is effective in reducing the costs related to influenza?

$\square$ Strongly agree
$\square$ Agree
$\square$ Do not agree/Do not disagree
$\square$ Disagree
$\square$ Strongly disagree

8. Do the administrators of your nursing home agree with the statement that HCWs have a special responsibility in preventing influenza transmission to residents?

$\square$ Strongly agree
$\square$ Agree
$\square$ Do not agree/Do not disagree
$\square$ Disagree
$\square$ Strongly disagree

9. Do the administrators of your nursing home agree with the statement that they have a moral responsibility to implement voluntary influenza vaccination of HCWs?

$\square$ Strongly agree
$\square$ Agree
$\square$ Do not agree/Do not disagree
$\square$ Disagree
$\square$ Strongly disagree

10. Do the administrators of your nursing home agree with the statement that mandatory influenza vaccination of HCWs should be implemented if voluntary vaccination fails to reach a sufficient vaccination rate?

$\square$ Strongly agree
$\square$ Agree
$\square$ Do not agree/Do not disagree
$\square$ Disagree
$\square$ Strongly disagree

11. Do you have any supplementary comments? 